\title{
OBSERVATIONS OF STELLAR OSCILLATIONS WITH THE HUBBLE SPACE TELESCOPE
}

\author{
David R. Soderblom \\ Space Telescope Science Institute \\ 3700 San Martin Drive \\ Baltimore, Maryland 21218 USA
}

\begin{abstract}
A rationale and plan for using the Hubble Space Telescope to observe solarlike oscillations in stars is presented.
\end{abstract}

\section{INTRODUCTION}

No confirmed detections yet exist of solar-like oscillations on another star. Such detections are technically demanding, requiring either extraordinary velocity resolution $\left(\sim \mathrm{cm} \mathrm{s}^{-1}\right)$ or high photometric precision $\left(S / N \gtrsim 10^{5}\right)$. There are two compelling reasons for attempting to observe stellar oscillations from space: 1) Removal of atmospheric effects, enabling instrument-limited detections. 2) The ability to stay on a target for an extended period, to achieve sufficient frequency resolution to see the low-degree splitting $(\ell=0$ vs. $\ell=2$ and $\ell=$ 1 vs. $\ell=3$ ), at the $10 \mu \mathrm{Hz}$ level. It is possible-perhaps probable-that ground-based observers will make a definitive detection of solar-like oscillations in the near future. It is unlikely, however, that anyone will detect the low-degree splitting, where some particularly significant physical information lies.

The Hubble Space Telescope (HST) is probably the first space instrument that is capable of detecting solar-like oscillations on other stars. No instrument on HST has sufficient velocity resolution, but the High Speed Photometer (HSP) should be capable of detecting the low-amplitude, white light variations produced by oscillations. A team of about 20 observers and theoreticians has been formed to define an observing program that exploits HST's capabilities, and to plan for the reduction, analysis, and interpretation of the results.

\section{GOALS}

1. To make a definitive detection of a solar-like oscillation on another star. This can firmly establish asteroseismology as a discipline, and provide a benchmark for groundbased observations. 
2. To test the claimed detection of oscillations in $\alpha$ Centauri A by Fossat, Grec, et al.. In particular, if the frequency spacing that they observe, $162 \mu \mathrm{Hz}$, is found to hold, there are difficulties with stellar structure models that are as serious as the solar neutrino problem.

3. To detect the low-degree mode splitting near $10 \mu \mathrm{Hz}$. This provides an indication of conditions at the very center of the star, and so is a check on how well the surface properties of a star indicate its evolutionary status.

4. To ultimately extend detections to a broader range of stars, as a means of testing the theory of stellar structure, and to delineate the basic properties of oscillations in late-type stars.

\section{TARGET SELECTION}

The ultimate goal of asteroseismology is the observational examination of the interior structures of late-type stars. Before that goal can be approached, a positive detection must be made and independently confirmed. We believe that HST is capable of making such a detection. Moreover, observations with HST can yield significantly greater physical information that ground-based data can.

But it is not sufficient to just make a detection, one must be able to interpret it as well. In other words, one needs to know the fundamental parameters of the star being observed. The most important of these is the star's mass. Therefore, the first criterion to be applied to a potential taget is that it lie in a nearby visual binary with a well-determined orbit. An additional benefit of observing stars in binaries is that the system's age can be determined from the stars' temperatures and luminosities.

Another important criterion is that the star resemble the Sun as much as possible. Until we know more about stellar oscillations, it seems prudent to take small steps. Other basic parameters of the star (temperature, radius, composition, rotation rate, etc.) should be known with reasonable precision.

For HST, a final criterion is that the star lie within the Continuous Viewing Zones (44.5 $\leq|\delta| \leq 78.5)$. At those declinations, stars can be observed for long periods with only minor interruptions due to passage through the South Atlantic Anomaly. This enables achieving very high frequency resolution, which is necessary for extracting the low-degree splitting.

All of these criteria point to exactly one star: $\alpha$ Centauri $A$. There is now probably no other star, save the Sun, whose properties are as well known. Recent refinements in the astrometry have led to accurate luminosities and masses for $\alpha$ Cen $A$ and $B$. The exceptional quality of data from the Coudé Echelle Spectrometer at the European Southern Observatory is now enabling studies of these stars' temperatures, composition, rotation rates, and levels of chromospheric activity. 


\section{OBSERVATIONAL PLAN}

Alpha Centauri A is very bright $(V=0.0)$. HSP's best performance is in its photoncounting mode, and in order to achieve a reasonable count rate ( $\lesssim 12 \mathrm{MHz})$ one needs to observe in the ultraviolet. We think that this is an advantage because in the far ultraviolet, the continuum is formed near the temperature minimum, which should respond like the chromosphere. On the Sun, the chromosphere shows higher amplitude oscillations that does the photosphere.

We wish to achieve $S / N \sim 10^{5}-10^{6}$, so that we need to count $\sim 10^{11}$ photons. At a counting rate of $\sim 10 \mathrm{MHz}$, about 20 hours are needed to detect the equivalent of the solar oscillations. We also intend to go beyond just a detection of the primary frequencies and their splittings, to reach the low-degree splitting at about the $10 \mu \mathrm{Hz}$ level. This will require sampling over a time of $\mathbf{4 0}$ to $\mathbf{5 0}$ hours, with this window being about half filled.

Can HST reliably detect a periodic signal with an amplitude of $10^{-5}$ or less? We believe so, but there are a number of subtle effects that can produce noise or a spurious signal. Most of these arise in the spacecraft guidance system, whose motions will result in photometric variations. Most of these effects take place on the time scales of the guidance system $(\leqslant 1$ sec) or orbital time scales (90 minutes), and so are well removed from the expected 5-minute periodicity. The amplitudes of these effects and any aliases should be determinable from test observations obtained during HST's Science Verification period. We believe that we can determine a realistic estimate of the level of detectability prior to the actual observations.

Extracting a periodic signal from a time series is generally a straightforward problem. We anticipate difficulties to arise from determining what the true stellar signal is, and from the possible spurious signals just mentioned. Along with the raw HSP data, we will examine the simultaneous HST engineering data to search for correlations between a photometric signal and instrument or spacecraft changes. The interpretation and modeling will proceed in a similar fashion, with independent efforts by several groups to reduce model dependence.

As this is written, the launch of HST is scheduled for December, 1988. Ground-based achievements in the next several years may well alter this plan. Our proposal team is listed below. 
HST Proposal Team for Stellar Seismology

\begin{tabular}{llc}
\hline \multicolumn{1}{c}{ Name } & \multicolumn{1}{c}{ Institution } & Country \\
\hline Sallie Baliunas & Center for Astrophysics & USA \\
Timothy Brown & High Altitude Obs. & USA \\
J. Christensen-Dalsgaard & Univ. of Aarhus & Denmark \\
Werner Däppen & High Altitude Obs. & USA \\
Pierre Demarque & Yale Univ. & USA \\
Eric Fossat & Observatoire de Nice & France \\
Søren Frandsen & Univ. of Aarhus & Denmark \\
Ingemar Furenlid & Georgia State Univ. & USA \\
Douglas Gough & Univ. of Cambridge & UK \\
D. B. Guenther & Yale Univ. & USA \\
Jack Harvey & National Solar Obs. & USA \\
Hugh Hudson & Univ. of Calif., San Diego & USA \\
Wolfgang Kalkofen & Center for Astrophysics & USA \\
Robert Kurucz & Center for Astrophysics & USA \\
A. Nordlund & Nordita & Denmark \\
Robert Noyes & Center for Astrophysics & USA \\
David Soderblom & Space Telescope Science Inst. & USA \\
Roger Ulrich & Univ. of Calif., Los Angeles & USA \\
Martin Woodard & Jet Propulsion Lab. & USA \\
\hline
\end{tabular}

